\title{
Do patients with rheumatoid arthritis have aorta stiffer than general population?

\author{
Petar Avramovski ${ }^{\mathrm{a}}$, Maja Avramovskac ${ }^{\mathrm{c}}$, Marija Arsovska - Nalbanti ${ }^{\mathrm{b}}$, \\ Pece Nikolovski ${ }^{\mathrm{e}}$, Emilija Sikole ${ }^{\mathrm{d}}$
}

\author{
${ }^{a}$ Clinical Hospital - Bitola, Departments of \\ Internal Medicine and Doppler \\ Ultrasonography, ${ }^{b}$ Clinical Hospital - Bitola, \\ Departments of Rheumatology and \\ Degenerative Arthropathie, 'University Clinic of \\ Obstetrics and Gynecology, University SS, \\ Cyril and Methodius, Skopje, ${ }^{\mathrm{I}}$ Institute of \\ Preclinical and Clinical Pharmacology with \\ Toxicology, University SS, Cyril and Methodius, \\ Skopje, ${ }^{e}$ Faculty of Economics - Prilep, \\ Department of Statistics, St. Celement of Ohrid \\ University, Bitola, Macedonia \\ Correspondence to Petar Avramovski, Docent \\ d-r Sci. med., MD, PhD, Department of Interna \\ Medicine, Clinical Hospital D-r Trifun Panovski' \\ Partizanska bb., 7000 Bitola, Macedonia. Tel: \\ +38970207 187, +38947253435; \\ e-mail: avramovski@gmail.com \\ Received 14 October 2017 \\ Accepted 4 December 2017 \\ The Egyptian Journal of Internal Medicine \\ 2018, 30:145-153
}

\begin{abstract}
Objective
The aim of this study was to compare the aortic stiffness in patients with rheumatoid arthritis (RA) with the aortic stiffness in general population (GP) and to evaluate the determinants of their stiffness.

Patients and methods

The study group consisted of 80 patients with RA and 71 matched GP. Pulse wave velocity (PWV) was assessed by Doppler ultrasonography. Biochemical analyses were conducted for serum using standard laboratory procedures.

Results

The mean PWV values were $7.687 \pm 1.03$ versus $7.253 \pm 0.97 \mathrm{~m} / \mathrm{s}$ in the patients with RA and GP, respectively. The average coordination of PWV and RA duration (RAD) variations is present by equation of linear regression: $y=7.1309+0.0971 x$. By multiple regression analysis, $C$-reactive protein, $\left(\beta_{\mathrm{st}}=0.1096, P=0.0009\right), \mathrm{RAD}$ $\left(\beta_{\mathrm{st}}=0.0917, \quad P=0.0018\right)$, high-density lipoprotein cholesterol $\left(\beta_{\mathrm{st}}=-1.0610\right.$, $P=0.0271)$, and cholesterol $\left(\beta_{\mathrm{st}}=0.8491, \quad P=0.0350\right)$ were independently associated with PWV in the patients with RA. C-reactive protein $\left(\beta_{\mathrm{st}}=0.1057\right.$, $P=0.0016)$, triglycerides $\left(\beta_{\mathrm{st}}=0.1730, \quad P=0.0117\right)$, low-density lipoprotein cholesterol $\left(\beta_{\mathrm{st}}=0.1467, P=0.0206\right)$, and cholesterol $\left(\beta_{\mathrm{st}}=0.0305, P=0.0261\right)$ were independently associated with PWV in the GP.

Conclusion

Arterial stiffness was more pronounced in the patients with RA than in the GP. The independent determinants of arterial stiffness in both groups include traditional risk factors. RAD is determinant in patients with RA only.
\end{abstract}

\section{Keywords: risk factors \\ Egypt J Intern Med 30:145-153 \\ (c) 2018 The Egyptian Journal of Internal Medicine \\ $1110-7782$}

aortic stiffness, Doppler ultrasonography, pulse wave velocity, rheumatoid arthritis, traditional

\section{Introduction}

Rheumatoid arthritis (RA) is a chronic progressive inflammatory disease, characterized by synovial inflammation and hyperplasia, leading to progressive cartilage and bone destruction [1]. RA is a disease with chronic systemic inflammation and immune system activation, which through early and enhanced effects on vascular function and morphology leads to accelerated atherosclerosis $[2,3]$. Chronic inflammation may impair vascular function and lead to an increase of arterial stiffness, an important determinant of cardiovascular risk [4]. Traditional cardiovascular risk factors such as hypertension, dyslipidemia, diabetes mellitus, smoking, obesity, and physical inactivity cannot entirely explain the higher level of cardiovascular complication, and there is growing evidence that chronic inflammation is the main culprit [5]. The standardized mortality ratio for patient with RA is 2.0 , and their median survival is as many as 17 years less than that of the general population (GP). Most of this excess mortality is attributable to cardiovascular events [6]. Mortality in patients with RA is higher than in the
$\mathrm{GP}$, which is mainly owing to premature cardiovascular disease. Accelerated atherosclerosis is not simply a lipid storage disease'. It is an inflammation that plays a major role in its initiation and progression, which attributes to chronic inflammation in patients with RA $[7,8]$.

The patients with atherosclerosis have increased arterial stiffness compared with patients without evident atherosclerosis. In the presence of atherosclerosis, stiffness of large arteries is increased, and it has been suggested that arterial stiffness may be a useful marker of the extent of atherosclerosis in the aorta [9]. Parameters that describe vessel stiffness include compliance and distensibility. The consequence of reduced compliance and distensibility is an increased propagation velocity of the pulse pressure along the arterial tree, called pulse wave velocity (PWV) which is inversely correlated with

This is an open access journal, and articles are distributed under the terms of the Creative Commons Attribution-NonCommercial-ShareAlike 4.0 License, which allows others to remix, tweak, and build upon the work non-commercially, as long as appropriate credit is given and the new creations are licensed under the identical terms. 
arterial distensibility and relative arterial compliance [10]. Decreased elastin, increased collagen in the arterial wall, and abnormal endothelial regulation of arterial smooth muscle tone are responsible for arterial stiffness in the aging process, diabetes mellitus, endstage renal disease, and RA [11]. In these groups, increased aortic stiffness, wave-reflection with consecutive elevation of PWV, and endothelial function are independent predictors of cardiovascular risk and may directly accelerate the atherosclerotic process [12].

PWV and augmentation index are the two major noninvasive methods of assessing arterial stiffness. PWV reflects the stiffness (elasticity) of segmental artery (carotid to femoral segment). It can be calculated as the distance $(D)$ traveled by the pulse wave divided by the time taken to travel the distance (T). Carotid-femoral PWV is considered to be the gold standard for assessing central arterial stiffness [13]. PWV as a measure in this study in patients with RA and GP represents the velocity of the pulse wave transit from the common carotid artery (CCA) to the femoral artery (FA). Thus, PWV is a measure of aortic stiffness, an elastic artery with muscular contributions to its compliance [14].

The aim of this study was to compare the aortic stiffness in patients with RA with GP and to evaluate the determinants of their stiffness.

\section{Patients and methods}

In this longitudinal observational study, we estimated 80 patients with RA older than 18 years who attended the rheumatology department of our public clinical hospital. All patients fulfilled the America College of Rheumatology diagnostic criteria for RA $[15,16]$. To avoid confounding by other risk factors for atherosclerosis, we used the following exclusion criteria: history of ischemic cardiovascular disease, valvular heart disease, cardiac arrhythmia, hypertension, renal impairment with reduced glomerular filtration rate $\left(\leq 60 \mathrm{ml} / \mathrm{min} / 1.73 \mathrm{~m}^{2}\right)$, diabetes mellitus (raised fasting blood glucose $\geq 7.0 \mathrm{mmol} / \mathrm{l}$ ), hyperlipidemia, premature menopause, recent surgery, pregnancy, and recent illness unrelated to RA. Approximately 93 (9.68\%) [9] of the beforeenrolled patients with RA were ineligible because of these exclusion criteria.

The control group consisted of 71 patients from the GP. The total number of participants recruited from ambulatory patients was equal to their proportion in the GP according to predefined criteria: age, sex, BMI, and smoking, compared with those of the study group patients. All the above-mentioned exclusion criteria were used during selection of the control group, too. All participants from both groups signed an informed consent, and Ethics Committee of our institution approved the study.

\section{Assessment}

Estimation of PWV

We used linear array $5-10 \mathrm{MHz}$ multifrequency ultrasound probe GE 10L (General Electric Logiq Pro 5, E Medical Systems, 4855 W Electric Avenue, Milwaukee, WI 53215, USA) at sequential Doppler signals recording of carotid and FA synchronized with electrocardiography (ECG) signal. First, we recorded the Doppler flow waveform of the proximal CCA and then of the distal site (FA) transcutaneously at the base of the neck for the left CCA and over the left FA in the groin. The next step is the detection of the delay or the difference in arrival time of the flow wave at these arterial locations. The basic principle of PWV estimation is shown by hand drawing (Fig. 1).

The distance line marked as D1 in Fig. 1 presents the traveling path that blood makes to access from the heart to the left CCA, and T1 line shows us the time needed for blood to cross the path. The distance line marked as D2 presents the traveling path that blood makes to access from the heart to the left FA, and T2 line shows us the time needed for blood to cross the path. The length of the time line $\mathrm{T} 1$ is determined by the distance between $\mathrm{R}$ wave of the ECG signal and the foot of the left CCA waveform signal. The length of the time line T2 is determined by the distance between $\mathrm{R}$ wave of the ECG signal and the foot of the left FA waveform signal. That means that synchronization start point for the both time lines (T1 and T2) is the same, i.e., R wave, (the second of three graphical deflections seen on typical ECG, Qwave, R wave, and S wave) which occurs in rapid succession named of QRS complex.

Distances between the sampling sites $\left(D=D_{2}-D_{1}\right)$ were measured as straight lines between the points on the body surface using a tape measure located at the same place as the ultrasound probe, with two-dimensional guidance to localize the exact position of the analyzed arterial site $[17,18]$. The pulse traveling time, or time delay $\Delta T$, was calculated by the equation $\Delta T=\mathrm{T}_{2}-\mathrm{T}_{1}$. The speed of the pulse wave $(V)$ was calculated with the standard equation for the speed (distance travelled per unit of time): $S(\mathrm{~m})=$ $V(\mathrm{~m} / \mathrm{s}) \times \Delta T$ or $\mathrm{PWV}=D / \Delta T$. On the basis of previous equation, aortic PWV was calculated as the ratio of distance $(D)$ to transit time $(\Delta T)$. 


\section{Clinical and biochemical parameters}

Clinical and biochemical parameters [plasma glucose, cholesterol, high-density lipoprotein cholesterol (HDL-C), low-density lipoprotein cholesterol (LDL-C), triglycerides, C-reactive protein (CRP), serum albumin, fibrinogen, and erythrocyte sedimentation rate $(\mathrm{ESR})]$ were determined in all participants using standard laboratory procedures, performed on a Cobas Mira S Analyzer (Roche Diagnostics, Holliston, Massachusetts, USA). Blood was drawn in a fasting state, and mean values obtained from three

\section{Figure 1}

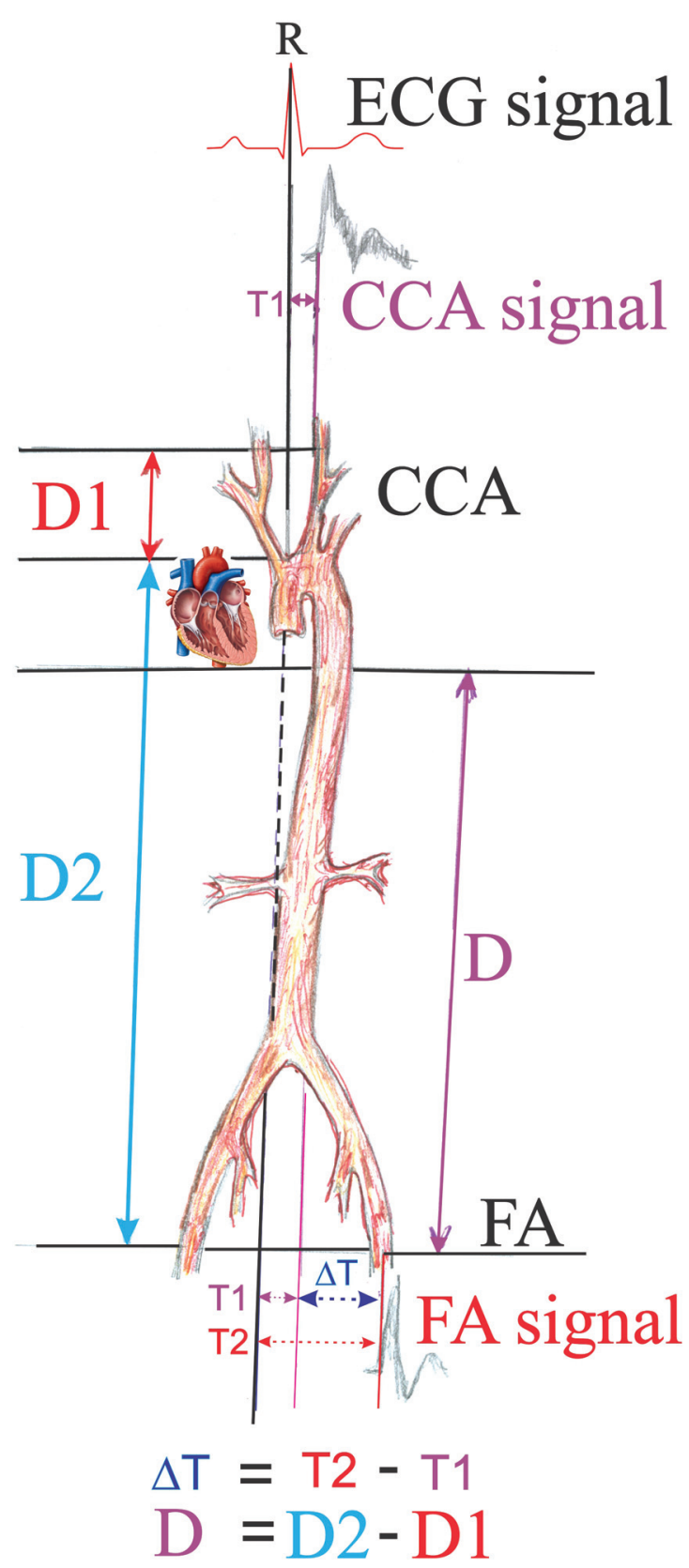

Pictorial basic principles of generating time diversity $(\Delta T)$ of ECG and carotid-femoral Doppler signal for pulse wave velocity estimation. CCA, common carotid artery; FA, femoral artery. measurements during the 3 months before PWV measurements were used in the analysis.

\section{Statistical analysis}

The data were analyzed using MedCalc for Windows, 13.0.6.0. (MedCalc Software, Ostend, Belgium). The results were expressed as mean $\pm \mathrm{SD}$ or percentage. Student's $t$-test for unpaired data was used to compare the patients with RA and GP. Simple linear regression analysis was performed to assess the associations between dependent and independent variables. Multiple backward regression analysis was used to predict outcome of a response variable. All tests were two sided, and $P$ less than 0.05 was considered to indicate a significant difference.

\section{Results}

During the 2-year period from May 2014 to April 2016, color Doppler ultrasonography measurement and biochemical and other demographic examination were successfully conducted on 80 patients with RA and $71 \mathrm{GP}$ (age: $45.8 \pm 8.78$ and $46.1 \pm 8.64$ years, respectively), with their BMI $23.43 \pm 2.72$ and 23.61 \pm 1.19 , respectively. The mean rheumatoid arthritis duration (RAD) was $6.2 \pm 3.9$ years. The demographic and biochemical characteristics of the patients are presented in Table 1.

The mean PWV in patients with RA was $7.687 \pm$ $1.03 \mathrm{~m} / \mathrm{s}$, and the mean PWV in GP was $7.253 \pm$ $0.97 \mathrm{~m} / \mathrm{s}$. There was a high statistical significance between the mean PWV in the patients with RA and GP $(P=0.0087)$. The results from unpaired $t-$ test between PWVs in both groups, mean, 95\% confidence interval (CI) of the mean, range, median, 25 th and 75 th percentiles, test statistic $t$, difference, $F$ test for equal variance, and two-way probability are presented in Fig. 2.

In patients with RA, we found the following coefficients of determination: $R^{2}=0.1176$ for relationship between PWV and RAD and $R^{2}=$ 0.3994 for relationship between PWV and age. The coefficient of determination $R^{2} \quad\left(0.1176=0.343^{2}\right)$ showed that $11.76 \%$ of the total variability was explained with the linear relation between PWV and RAD accompanied by other determinants, or that $11.76 \%$ from PWV (as measure of aortic stiffness) was dependent on RAD as the predictor and other determinants (glucose, cholesterol, HDL-C, LDL-C, $\mathrm{CRP}$, fibrinogen, and so on). The coefficient of determination between PWV and RAD in young individuals (mean age: $37.3 \pm 2.1$ years and mean 
Table 1 Demographic and biochemical characteristics of the patients

\begin{tabular}{|c|c|c|c|}
\hline Characteristics & $\begin{array}{l}\text { Patients with RA } \\
\qquad(N=80)\end{array}$ & $\begin{array}{c}\text { GP } \\
(N=71)\end{array}$ & $P$ value \\
\hline Age (years) & $45.8 \pm 8.78$ & $46.1 \pm 8.64$ & 0.833 \\
\hline Female $[n(\%)]$ & $62(77.5)$ & $55(77.46)$ & 0.995 \\
\hline Weight (kg) & $63.26 \pm 9.72$ & $\begin{array}{l}65.26 \\
\pm 7.12\end{array}$ & 0.156 \\
\hline Height $(\mathrm{cm})$ & $164.27 \pm 5.92$ & $\begin{array}{l}166.23 \\
\pm 8.45\end{array}$ & 0.098 \\
\hline BMI $\left(\mathrm{kg} / \mathrm{m}^{2}\right)$ & $23.43 \pm 2.72$ & $\begin{array}{l}23.61 \\
\pm 1.19\end{array}$ & 0.607 \\
\hline PWV (m/s) & $7.687 \pm 1.03$ & $\begin{array}{l}7.253 \\
\pm 0.97\end{array}$ & 0.009 \\
\hline Glucose $(\mathrm{mmol} / \mathrm{l})$ & $5.39 \pm 0.51$ & $5.30 \pm 0.62$ & 0.329 \\
\hline Smokers [n (\%)] & 17 (21.25) & $22(30.98)$ & 0.175 \\
\hline $\begin{array}{l}\text { Cholesterol } \\
(\mathrm{mmol} / \mathrm{l})\end{array}$ & $4.71 \pm 0.53$ & $5.28 \pm 0.84$ & $<0.0001$ \\
\hline $\mathrm{HDL}-\mathrm{C}(\mathrm{mmol} / \mathrm{l})$ & $1.04 \pm 0.35$ & $1.06 \pm 0.29$ & 0.705 \\
\hline LDL-C (mmol/l) & $2.90 \pm 0.61$ & $3.09 \pm 0.72$ & 0.081 \\
\hline $\begin{array}{l}\text { Triglycerides } \\
(\mathrm{mmol} / \mathrm{l})\end{array}$ & $1.64 \pm 0.74$ & $1.31 \pm 0.97$ & 0.019 \\
\hline CRP (mg/l) & $5.31 \pm 2.06$ & $3.57 \pm 1.94$ & $<0.0001$ \\
\hline Albumin (g/l) & $34.2 \pm 7.1$ & $46.9 \pm 5.7$ & $<0.0001$ \\
\hline Fibrinogen (g/l) & $3.56 \pm 1.32$ & $2.57 \pm 0.74$ & $<0.0001$ \\
\hline $\operatorname{ESR}(\mathrm{mm} / \mathrm{h})$ & $23.4 \pm 18.4$ & $12.4 \pm 5.7$ & $<0.0001$ \\
\hline
\end{tabular}

CRP, C-reactive protein; ESR, erythrocyte sedimentation rate; GP, general population HDL-C, high-density cholesterol; LDL-C, lowdensity cholesterol; PWV, pulse wave velocity; RA, rheumatoid arthritis.

RAD: $4.9 \pm 2.7$ years) was $R^{2}=0.4502$ (or $0.671^{2}$ ). This shows that $45.02 \%$ of the total variability was explained with the linear relation between PWV and RAD in young individuals, or that $45.02 \%$ from PWV was dependent of RAD as the predictor. The coefficient of determination $R^{2}\left(0.3994=0.632^{2}\right)$ showed that $39.94 \%$ of the total variability was explained with the linear relation between PWV and age accompanied by other determinants, or that $39.94 \%$ from PWV was dependent on age of the patients with $\mathrm{RA}$ as the predictor and $60.06 \%$ from the other determinants (glucose, cholesterol, HDL-C, LDL$\mathrm{C}, \mathrm{CRP}$, fibrinogen, and other traditional and nontraditional risk factors).

In GP, we found coefficients of determination: $R^{2}=0.6320$ for relationship between PWV and age. The coefficient of determination $R^{2}\left(0.6320=0.795^{2}\right)$ showed that $63.20 \%$ of the total variability was explained with the linear relation between PWV and age in GP accompanied by other determinants. That means that 63.20\% from PWV was dependent on age of the GP as the predictor and $36.80 \%$ from the other aforementioned determinants (glucose, cholesterol, HDL-C, and so on). The summary scatter plot diagram of relationship between PWV and other determinants (RAD and age) in patients with RA and GP is shown in Fig. 3.
Figure 2

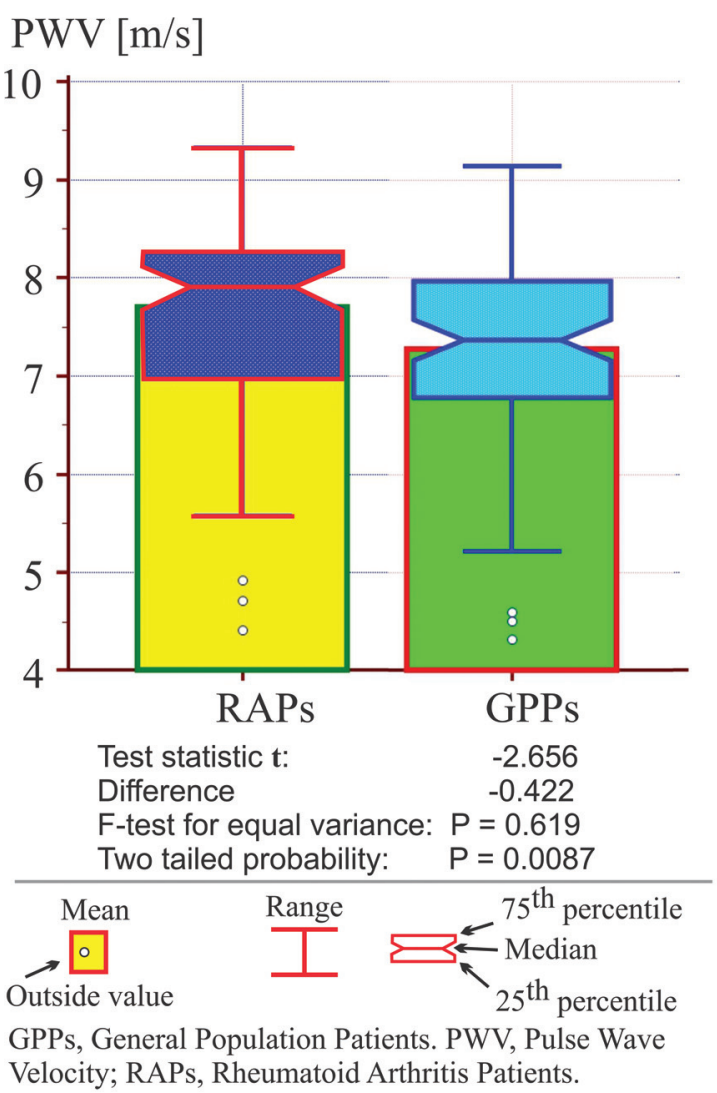

Test for unpaired data between the patients with rheumatoid arthritis and general population. PWV, pulse wave velocity.

Figure 3 shows a scatter plot of PWV and other variables: RAD and age in patients with RA and age in GP. Each circle has the value of one variable determining the position on the horizontal axis (RAD and age in patients with RA, and age in GP) and the value of the other variable (PWV) determining the position on the vertical axis. There was a positive association between PWV and these variables. We can compare the slopes (steepness of the angle of the regression line) of the regression line to estimate the overall strength of the relationship. The first plot shows a modest positive relationship between variables (PWV and $\mathrm{RAD}$ ), the second plot shows a stronger positive association between variables (PWV and age of patients with RA), and third plot shows strongest positive association between PWV and age in GP.

\section{Linear regression}

The results of linear regression represent the relationship between a scalar dependent variable $Y(\mathrm{PWV}, \mathrm{m} / \mathrm{s})$ and an explanatory variable denoted $X$ (RAD). They were presented as equation of simple linear regression $y=7.1309+0.0971 x \quad(P<0.0001$, intercept; $P=0.0018$, slope), regression parameter $b_{0}=7.1309$, regression parameter $b_{1}=0.0971$, and coefficient of determination 


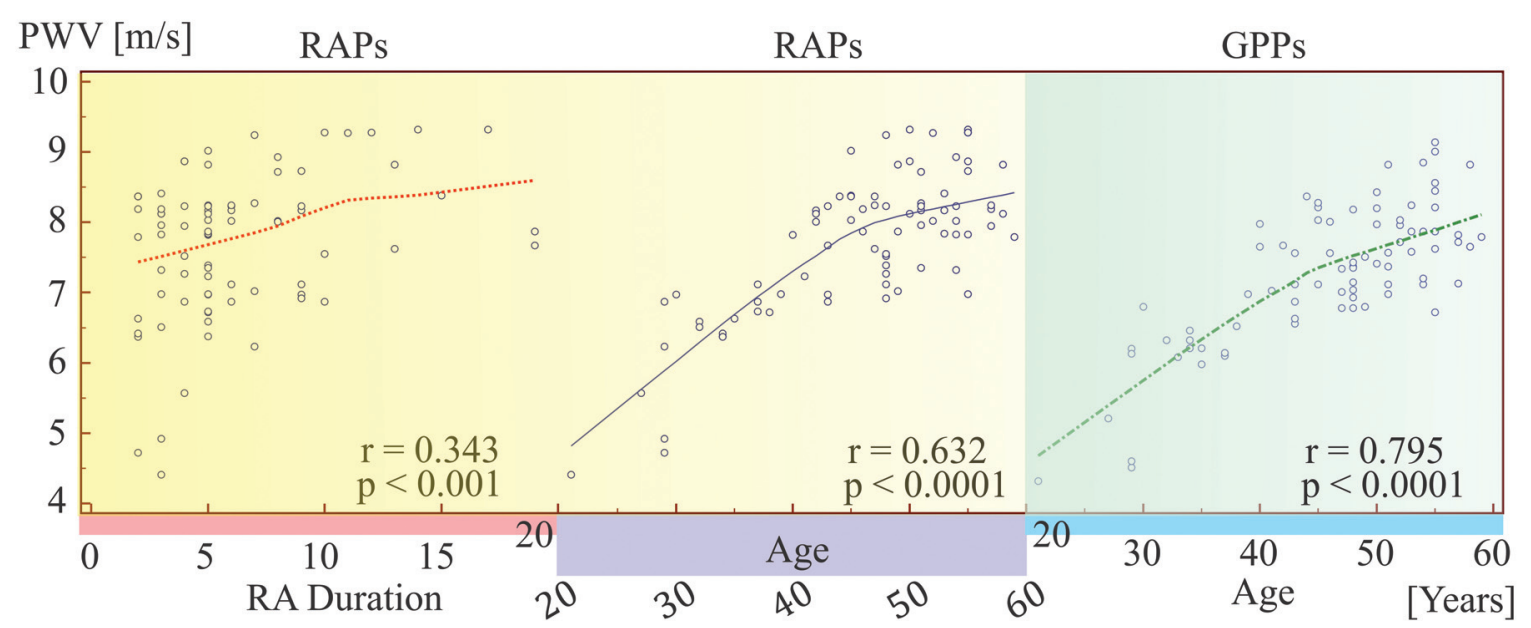

GPPs, General Population Patients; PWV, Pulse Wave Velocity; $p$ - coefficient of significance; $\mathrm{r}$ - coefficient of correlation; RA Duration, Rheumatoid Arthritis Duration; RAPs, Rheumatoid Arthritis Patients.

Summary scatter plot diagram of relationship between pulse wave velocity (PWV) and rheumatoid arthritis duration (RA duration), PWV and age in patients with rheumatoid arthritis, and PWV and age in general population individuals. $P$, coefficient of significance; $r$, coefficient of correlation.

$R^{2}=0.1176$. The equation of simple linear regression showed the average coordination of PWV and RAD variations. With this equation, we obtained the evaluated (theoretical) PWV values to compare with its empirical values. The regression parameter $b_{0}=7.1309$ showed the expected theoretical value of PWV in case that $\mathrm{RAD}$ would have a value equal to zero. This parameter also showed the point of the $y$-axis (dependent variable axis, PWV) through which the regression line passed. The regression parameter $b_{1}=0.0971$ signified that with each increase of one unit (year) in RAD, the PWV score increased by $0.0971 \mathrm{~m} / \mathrm{s}$ or $9.71 \mathrm{~cm} / \mathrm{s} \quad(0.971 \mathrm{~m} / \mathrm{s} /$ decade). The coefficient of determination $R^{2}(0.1176)$ showed that $11.76 \%$ of the total variability was explained with the linear relation between $\mathrm{PWV}_{[\mathrm{m} / \mathrm{s}]}$ and $\mathrm{RAD}_{[\text {year] }}$ or that $11.76 \%$ from PWV was dependent on RAD. Only $11.76 \%$ of the changes in PWV were the result of RAD value changes, and the remaining from the total variability between them were not explained (88.24\% of PWV were dependent on other factors, which were not covered with the regression model).

Figure 4 shows a scatter plot of PWV and RAD. The data from each of the 80 patients with RA were displayed as a collection of blue circles determining the PWV and RAD. Each point had the value of one variable (RAD) determining the position of the $x$ (horizontal axis) and the value of the other variable (PWV) determining the position on the $y$ (vertical axis).

There was a positive association between these variables, showed by the thickened red line.
Figure 4

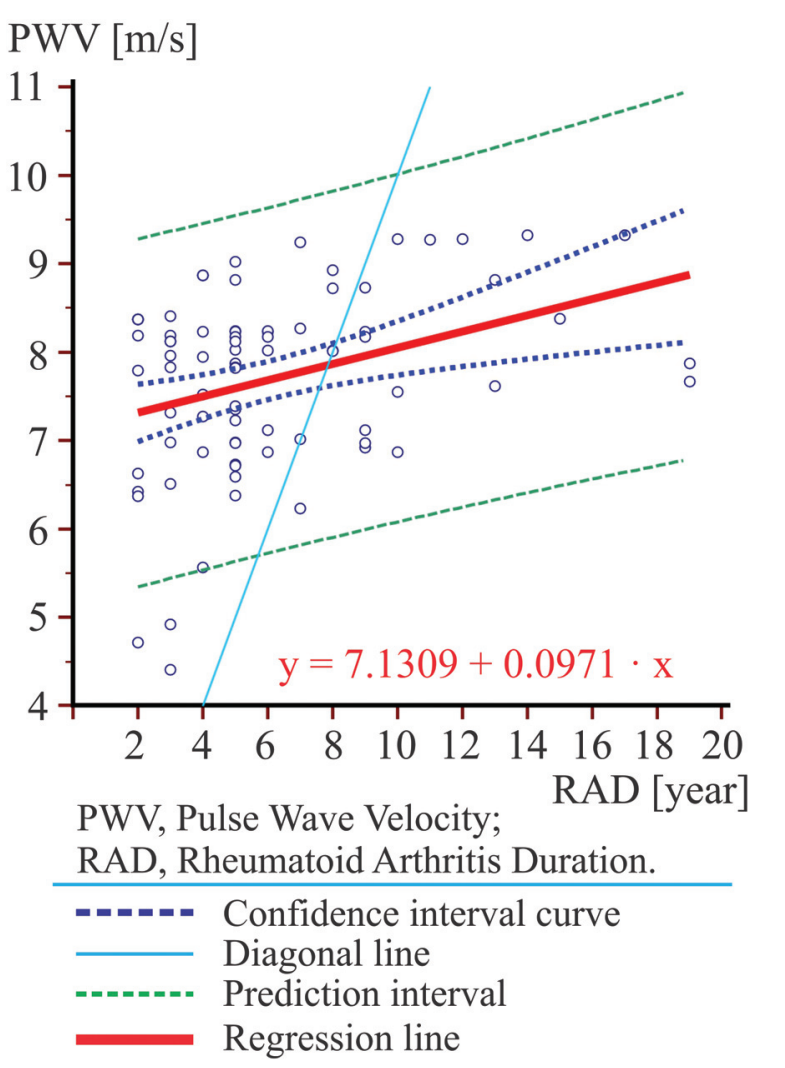

Scatter plot of pulse wave velocity (PWV) and rheumatoid arthritis duration (RAD).

The 95\% CI of intercept (95\% CI=6.718-7.544) is shown by the blue dashed line, and prediction interval is shown by the green dashed line. The diagonal line (blue thin line) is a reference line $(x=y)$ 
that is splitting the graph in two equal areas by straight of $45^{\circ}$.

\section{Multiple regression}

Assessments [standardized coefficient $\beta\left(\beta_{\text {st }}\right)$, SE of $\beta_{\mathrm{st}}, t$, and $P$ value] of the independent predictor CRP or determinants (RAD, HDL-C, cholesterol, triglycerides, $\mathrm{LDH}-\mathrm{C}$, and cholesterol) for increasing of PWV as dependent variable $Y$ in patients with RA and GP after backward multiple regression analysis are shown in Table 2.

The $P$ values showed the following order of statistical significance: CRP (0.0009), RAD (0.0018), HDL-C (0.0271), and cholesterol (0.0350) for patients with RA, and CRP (0.0016), triglycerides (0.0117), LDL-C (0.0206), and cholesterol (0.0261) for GP. There was an inverse correlation (negative $\beta_{\text {st }}$ coefficient, $\left.\beta_{\mathrm{st}}=-1.0610\right)$ between the PWV and HDL-C in patients with RA only.

\section{Discussion}

There are a small number of studies that examined aortic stiffness in patients with RA and compared their results with the results of aortic stiffness in the GP [19]. Most of the studies that have evaluated the aortic stiffness expressed by PWV emphasized only the cardiovascular mortality risk factors in patients with RA and have not paid attention to aortic stiffness for comparison between the groups, especially not the factors that determine it $[20,21]$.

We compared the aortic stiffness in 80 patients with RA and 71 matched controls and evaluated the determinants of their aortic stiffness. We found a statistically significant difference between aortic PWVs as measure of aortic stiffness in the patients with RA and the GP $(P=0.009)$. Although we selected younger patients in the patients with RA and GP groups with aim to eliminate the effect of the natural process of atherosclerosis during ageing, the differences in stiffness of the aorta were still evident with statistical significance. It is obvious that these differences are generated by some other processes besides ageing in patients with RA, processes which further contribute to the advanced progression of arterial stiffness in patients with RA. Because PWV is an age-dependent parameter, the clinicians need to know the mean value of PWV in GP. The age-adjusted mean PWV values in the GP in our study match with the mean PWV values in GP in another study (our results: $7.253 \pm 0.97 \mathrm{~m} / \mathrm{s}$, vs. range of another study results: $6.68-7.03 \mathrm{~m} / \mathrm{s}$ ) [22].

We found that the mean PWV values in patients with $\mathrm{RA}$ were slightly different because of the different ages in our patients with RA group $(7.687 \mathrm{~m} / \mathrm{s}$, in 45.8 year vs. $8.35 \mathrm{~m} / \mathrm{s}$ in 57 years) from those in the study by Mäki-Petäjä et al. [23]. A systematic review and metaanalysis of literature studies about arterial stiffness in 1472 patients with RA and 1583 controls found significantly higher aortic PWV in patients with RA than in controls (mean difference: $1.32 \mathrm{~m} / \mathrm{s}$, $P<0.00001)$. The smaller mean difference of PWV between RA and controls in our study resulted from younger age (45-46 years) of the studied population than the patients from systematic reviews of literature studies by Ambrosino et al. [24].

Despite the effect of the common traditional risk factors for atherosclerosis, increased aortic PWV or reduced arterial elasticity in patients with RA compared with lower PWV in GP is occurring independently of traditional risk factors.

Table 2 Multiple backward regression analysis of determinants of pulse wave velocity in patients with rheumatoid arthritis and general population

\begin{tabular}{|c|c|c|c|c|c|}
\hline & \multicolumn{5}{|c|}{ Multiple regression Dependent Y; PWVMethod: BackwardEnter: if $P<0.1$; remove: if $P>0.3$} \\
\hline & Independent variables & $\beta_{\text {st }}$ coefficient & SE & $t$ & $P$ \\
\hline \multirow[t]{5}{*}{ Patients with RA } & CRP & 0.1096 & 0.0316 & 3.4720 & 0.0009 \\
\hline & $\mathrm{RAD}$ & 0.0917 & 0.0284 & 3.2260 & 0.0018 \\
\hline & HDL-C & -1.0610 & 0.4707 & -2.2540 & 0.0271 \\
\hline & Cholesterol & 0.8491 & 0.3954 & 2.1480 & 0.0350 \\
\hline & \multicolumn{5}{|c|}{ Variables not included in the model: albumin, LDL-Ch, TG, fibrinogen, glucose, and ESR } \\
\hline \multirow[t]{5}{*}{ GP } & CRP & 0.1057 & 0.0322 & 3.2830 & 0.0016 \\
\hline & Triglycerides & 0.1730 & 0.0667 & 2.5930 & 0.0117 \\
\hline & LDL-C & 0.1467 & 0.0621 & 2.3640 & 0.0206 \\
\hline & Cholesterol & 0.0305 & 0.0134 & 2.2760 & 0.0261 \\
\hline & \multicolumn{5}{|c|}{ Variables not included in the model: albumin, HDL-C, fibrinogen, glucose, and ESR } \\
\hline
\end{tabular}

$\beta_{\text {st }}, \beta$ standardized; CRP, C-reactive protein; ESR, erythrocyte sedimentation rate; GP, general population; HDL-C, high-density lipoprotein cholesterol; LDL-C, low-density lipoprotein cholesterol; PWV, pulse wave velocity; RA, rheumatoid arthritis; RAD, rheumatoid arthritis duration. 
The patients with RA showed higher aortic PWV than GP on the same age, but whether increased PWV is a consequence of RA or reflects vascular disease is unknown. In the GP group, only traditional risk factors (hypertension, diabetes mellitus, dyslipidemia, smoking, obesity, and physical inactivity) associated with progression of arterial stiffness in the elderly were evident. Unlike them, in patients with RA, arterial stiffness (i.e. PWV) is accelerated owing to synergism between age and traditional risk factors plus factors related to the RA (i.e., nontraditional risk factors: inflammatory markers CRP, ESR and serum albumin, disease activity scores, seropositivity, physical disability, destructive changes on joint radiographs, extra-articular manifestations, and corticosteroid use) [25]. That is the reason which suggests that classic risk factors do not explain excess vascular disease in patients with RA [2].

In the patients with RA, by coefficients of determination we found that ageing process is still the leading factor in the genesis of atherosclerosis and blood vessels hardening, but the effect of this process is less pronounced than the effect of ageing on artery stiffness in GP (39.94 vs. 63.20\%, respectively). The overall strength of the relationship between PWV and RA duration and between PWV and age has been presented by different slopes in the summary scatter plot. The effect of RAD on blood vessels hardening is smaller, but it has its own meaning (11.76\%). In young individuals where the process of atherosclerosis is not yet advanced, the RAD participation showed stronger effect on artery stiffness genesis than natural ageing process in patients with RA (45.02\%). Using linear regression equation, we found that patients with RA have PWV increase of $97.1 \mathrm{~cm} / \mathrm{s}(0.971 \mathrm{~m} / \mathrm{s}$ or $\sim 1 \mathrm{~m} / \mathrm{s})$ per decade of RAD. Tomlinson and colleagues proved that increase in PWV of $1 \mathrm{~m}$ is equate to a $39 \%$ increased risk of cardiovascular events, and that data should not be underestimated $[18,26]$. A lot of studies provide evidence that RA is associated with increase in arterial stiffness as assessed by noninvasive PWV measurement. The vascular stiffness, as measured by PWV, predominantly reflects the stiffness of large- and medium-sized arteries. This suggests that a higher degree of stiffness of medium-sized conduit arteries may play a role in protecting the microcirculation from a highly pulsatile forward pressure wave $[4,27]$.

Abnormalities in these arteries may result from functional abnormalities, such as endothelial dysfunction, to structural changes, such as atherosclerosis [28]. The patients with RA who had no cardiovascular symptoms showed impaired diastolic function indicating that diastolic dysfunction is one of the first detectable cardiovascular abnormalities in this disease, as an accompanying sign of the increased PWV. Systolic function is conveniently (although not always accurately) measured as the ejection fraction. In patients with heart failure, the stage of diastolic dysfunction is a stronger predictor of mortality than ejection fraction $[29,30]$.

Considering the results obtained by linear regression analysis that $60 \%$ of PWV change in patients with RA and $37 \%$ of PWV change in GP are dependent on additional (not ageing) traditional and nontraditional risk factors, we made multiple regression analysis to find their determinants and predictors. By multiple regression analysis, we found determinants of stiffness progression (PWV) expressed by $\beta_{\mathrm{st}}$ coefficients: CRP, $\mathrm{RAD}, \mathrm{HDL}-\mathrm{C}$, and cholesterol. We found inverse correlation between $\mathrm{PWV}$ and $\mathrm{HDL}-\mathrm{C}$ in patients with RA. Patients with RA have accelerated atherosclerosis despite the appearance of having a less atherogenic lipid profile than GP. The major activities of HDL-C are its antioxidant and cholesterol efflux function. HDL-C antioxidant capacity inversely associated with inflammation and RA disease activity [31]. Patients with RA demonstrate low total cholesterol driven mainly by low HDL-C concentrations. Numerous studies report an inverse correlation between inflammatory markers (CRP and ESR) and HDL-C. Despite the statistically high significant difference between laboratory markers (cholesterol, albumin, fibrinogen, and ESR), we did not find any statistically significant associative connection of the aforementioned variables with PWV. Traditional cardiovascular risk factors certainly contribute to the increased risk of mortality in patients with RA, but do not fully explain it [32]. Rather, the high systemic inflammatory burden associated with RA appears to be a key driver of increased artery stiffness, increased PWV, and cardiovascular risk. The elevated levels of cytokines and other inflammatory mediators detected in RA, like $\mathrm{CRP}$, are included in the concept of inflammatory-driven atherogenesis and artery stiffness progression [2]. In patients with RA, the primary site of inflammation is the synovial tissue, from which cytokines can be released into the systemic circulation. Serum albumin concentration, although it was low in most patients with RA and in our study too, did not correlate significantly with PWV. The backward model of multiple regression analysis excluded this variable from the model.

\section{Study limitations}

The first limitation of this study was the relative small number of sampled patients. Recruiting female and 
especially male patients in sufficient numbers certainly will improve the significance in the study results. Considering the fact that selecting a sufficient number of patients of young age who are spared from the natural process of the atherosclerosis and with no additional comorbidities is very difficult, we that the number of included patients is not that small after all.

Using linear regression analysis instead of nonlinear regression model to assess the association between dependent and independent variables (PWV and RAD) during stiffening and atherosclerotic processes that are not linear is another limitation of our study. Yet, we decided to use this approximation because the small interval of the average lasting sickness from just 6 years belongs to the curve segment of the linear part of the exponential nonlinear process of stiffening and aging of the blood vessels, whether as an atherosclerosis process or from RA.

\section{Conclusion}

We conclude that classic and novel risk factors that can be significantly influenced by the systemic inflammation in RA (CRP, cholesterol) rather than cholesterol or high blood pressure alone will better predict increases in arterial stiffness and PWV. The PWV is significantly pronounced in patients with RA than PWV in GP, even in young patients with RA, before the first signs of atherosclerosis occur. CRP, RAD, and cholesterol are determinants with an effect on the PWV increase and HDL-C as determinant with an effect on stiffness decrease. The effect of RAD, with slightly lower value of ' $P$ ' than the $P$ value of CRPs, is just another proof that RA duration affects the blood vessels making it more rigid. It makes the $\mathrm{RAD}$ to be the leading determinant in the increase of PWV and vascular stiffness.

\section{Financial support and sponsorship}

Nil.

\section{Conflicts of interest}

There are no conflicts of interest.

\section{References}

1 Bazzichi L, Ghiadoni L, Rossi A, Bernardini M, Lanza M, De Feo F, et al. Osteopontin is associated with increased arterial stiffness in rheumatoid arthritis. Mol Med 2009; 15:402-406.

2 Sattar N, McCarey DW, Capell H, McInnes IB. Explaining how 'high-grade' systemic inflammation accelerates vascular risk in rheumatoid arthritis. Circulation 2003; 108:2957-2963.

3 Veldhuijzen van Zanten JJ, Kitas GD. Inflammation, carotid intima-media thickness and atherosclerosis in rheumatoid arthritis. Arthritis Res Ther 2008; 10:102.
4 Klocke R, Cockroft JR, Taylor GJ, Hall IR, Blake DR. Arterial stiffness and central blood pressure, as determined by pulse wave analysis, in rheumatoid arthritis. Ann Rheum Dis 2003; 62:414-418.

5 Pieringer $\mathrm{H}$, Pichler M. Cardiovascular morbidity and mortality in patients with rheumatoid arthritis: vascular alterations and possible clinical implications. QJM 2011; 104:13-26.

6 Wong M, Toh L, Wilson A, Rowley K, Karschimkus C, Prior D, et al. Reduced arterial elasticity in rheumatoid arthritis and the relationship to vascular disease risk factors and inflammation. Arthritis Rheum 2003; 48:81-89.

7 Aviña-Zubieta JA, Choi HK, Sadatsafavi M, Etminam M, Esdale JM, Lacaill D. Risk of cardiovascular mortality in patients with rheumatoid arthritis: a meta-analysis of observational studies. Arthritis Rheum 2008; 59:1690-1697.

8 del Rincón ID, Williams K, Stern MP, Freeman GL, Escalante A. High incidence of cardiovascular events in rheumatoid arthritis cohort not explained by traditional cardiac risk factors. Arthritis Rheum 2001; 44:2737-2745.

9 Tanaka H, Dinenno FA, Monahan KD, Clevenger CM, DeSouza CA, Seals DR. Aging, Habitual exercise, and dynamic arterial compliance. Circulation 2000; 102:1270-1275.

10 Cecelja M, Chowienczyk P. Role of arterial stiffness in cardiovascular disease. JRSM Cardiovasc Dis 2012; 1:10-17.

11 Zieman SJ, Melenovsky V, Kass DA. Mechanisms, pathophysiology, and therapy of arterial stiffness. Arterioscler Thromb Vasc Biol 2005; 25:932-943.

12 Vizzardi E, Cavazzana I, Ceribelli A, Tincani A, Dei Cas L, Franceschini F. Aortic stiffness and left ventricular hypertrophy in rheumatoid arthritis: comment on the article by Rudominer, et al. Arthritis Rheum 2009; 60:2852-2853.

13 Laurent S, Cockcroft J, Van Bortel L, Boutouyrie P, Giannattasio C, Hayoz D, et al. Expert consensus document on arterial stiffness: methodological issues and clinical applications. Eur Heart J 2006; 27:2588-2605.

14 Cohn JN. Arterial stiffness, vascular disease, and risk of cardiovascular events. Circulation 2006; 113:602-603.

15 Hua C, Daien Cl, Combe B, Landewe R. Diagnosis, prognosis and classification of early arthritis: results of a systemic review informing the2016 update of the EULAR recommendations for the management of early arthritis. RMD Open 2017; 3:40-46.

16 Aletaha D, Neogi T, Silman AJ, Funovits J, Felson DT, Bingham OC, et al. 2010 Rheumatoid Arthritis Classification Criteria. Arthritis Rheum 2010; 62:2569-2581.

17 Avramovski P, Janakievska P, Koneska M, Sotiroski K, Sikole A. Asssociation between pulse wave velocity, aortic vascular calcification, and bone mineral density in chronic hemodialysis patients and general population. ISRN Vasc Med 2013; 10: 1-9.

18 Avramovski $\mathrm{P}$, Janakievska $\mathrm{P}$, Sotiroski K, Sikole A. Accelerated progression of arterial stiffness in dialysis patients compared with the general population. Korean J Intern Med 2013; 28:464-474.

19 Sliem H, Nasr G. Change of the aortic elasticity in rheumatoid arthritis: Relationship to associated cardiovascular risk factors. J Cardiovasc Dis Res 2010; 1:110-115.

20 Kaplan MJ. Cardiovascular complications of rheumatoid arthritis: assessment, prevention, and treatment. Rheum Dis Clin North Am 2010; 36:405-426.

21 Roman MJ, Salmon JE. Cardiovascular manifestation of rheumatologic diseases. Circulation 2007; 116:2346-2355.

22 Diaz A, Galli C, Tringler M, Ramirez A, Cabrere Fischer El. Reference values of pulse wave velocity in healthy people from an urban and rural Argentinean population. Int J Hypertens 2015; 2015:983928.

23 Mäki-Petäjä KM, Hall FC, Booth AD, Wallace SM, Yasmin xxxx, Bearcroft $\mathrm{PW}$, et al. Rheumatoid arthritis is associated with increased aortic pulsewave velocity, which is reduced by anti-tumor necrosis factor-alpha therapy. Circulation 2006; 114:1185-1192.

24 Ambrosino P, Tasso M, Lupoli R, Di Minno A, Baldassare D, Tremoli E, et al. Non-invasive assessment of arterial stiffness in patients with rheumatoid arthritis: a systematic review and meta-analysis of literature studies. Ann Med 2015; 47:457-467.

25 Jennifer KP, Tobias $\mathrm{P}$, Jing M, JoAnn EM, Susan EH, Kaumudi J, et al. Inflammatory markers and the risk of coronary heart disease in men and women. N Engl J Med 2004; 351:2599-2610. 
26 Tomlinson L, Ben-Shlomo Y, Caplin B, Delles C, Ferro CJ, Goddard J, et al. Renal disease is associated with accelerated vascular ageing: initial results of the UK research alliance into kidney disease and arterial stiffness (Ureka) collaboration. J Hypertens 2010; 28:e417.

27 Fortier C, Agharazi M. Arterial stiffness gradient. Pulse (Basel) 2016 3:159-166.

28 Wilkinson IB, Qasem A, McEniery CM, Webb DJ, Avolio AP, Cockcroft JR Nitric oxide regulates local arterial distensibility in vivo. Circulation 2002; 105:213-217.
29 Fagard R, Pardaens K. Left ventricular diastolic function predicts outcome in uncomplicated hypertension. Am J Hypertens 2001; 14:504-508.

30 Kitzman DW, Little WC. Left ventricle diastolic function and prognosis. Circulation 2012; 125:743-745.

31 Ormseth MJ, Stein CM. High-density lipoprotein function in rheumatoid arthritis. Curr Opin Lipidol 2016; 27:67-75.

32 Dessein PH, Joffe BI, Veller MG, Stevens BA, Tobias M, ReddiK, StanwixAE. Traditional and nontraditional cardiovascular risk factors are associated with atherosclerosis in rheumatoid arthritis. J Rheumatol 2005; 32:435-442. 